\title{
Cytochrome $c$ modulates the mitochondrial signaling pathway and polymorphonuclear neutrophil apoptosis in bile duct-ligated rats
}

\author{
XUESONG DENG ${ }^{1,2}$, TONGMING DENG ${ }^{3}$, YONG NI $^{2}$, YONGQIANG ZHAN ${ }^{2}$, \\ WENLONG HUANG $^{2}$, JIANFENG LIU ${ }^{2}$ and CAIXIAN LIAO ${ }^{1}$ \\ ${ }^{1}$ Department of Hepatobiliary Surgery, Nanfang Hospital, Southern Medical University, Guangzhou, Guangdong 510515; \\ ${ }^{2}$ Department of Hepatobiliary Surgery, The Second People's Hospital of Shenzhen \\ (The First Affiliated Hospital of Shenzhen University), Shenzhen, Guangdong 518035; \\ ${ }^{3}$ Department of General Surgery, Baoan Central Hospital of Shenzhen, \\ Shenzhen, Guangdong 518102, P.R. China
}

Received December 11, 2014; Accepted February 4, 2016

DOI: $10.3892 /$ etm.2016.3313

\begin{abstract}
It has been observed that polymorphonuclear neutrophils (PMN) increase in number and function during obstructive jaundice (OJ). However, the precise mechanisms underlying PMN apoptosis during OJ remain poorly understood. The aim of the present study was to investigate the modulation of cytochrome $c$ (Cytc) on the mitochondrial signaling pathway in bile duct-ligated (BDL) rats and the effect on PMN apoptosis following the intravenous administration of Cytc. Rats were randomly divided into four groups: A control group, a sham group, a BDL group and a BDL + Cytc group (rats with common bile duct ligation as well as Cytc intravenous injection). Blood samples were collected from the inferior vein cava for biochemical analysis and separation of the PMN. PMN apoptosis was evaluated using flow cytometry. The mitochondrial membrane potential $(\Delta \Psi \mathrm{m})$ of PMN was detected by rhodamine-123 staining. The Cytc protein expression levels were examined using western blotting. PMN mitochondria were observed using transmission electron microscopy. The results of the present study revealed that the PMN apoptosis rate in rats decreased gradually from 12 to $72 \mathrm{~h}$ following BDL to levels that were significantly lower than those of the control group and the sham group. Compared with the corresponding time point of the BDL group, the BDL + Cytc group showed a significantly increased PMN apoptosis rate. The mean fluorescence intensity (MFI) of $\Delta \Psi \mathrm{m}$ decreased from 12 to $72 \mathrm{~h}$ following BDL, and was significantly increased compared with the control and sham groups. MFI in the BDL + Cytc
\end{abstract}

Correspondence to: Professor Caixian Liao, Department of Hepatobiliary Surgery, Nanfang Hospital, Southern Medical University, 188 Guangzhou North Avenue, Guangzhou, Guangdong 510515, P.R. China

E-mail: 3275990267@qq.com

Key words: cytochrome $c$, polymorphonuclear neutrophils, apoptosis, mitochondria, apoptotic pathway group was higher compared with that in the BDL group. Cytc expression levels increased in the mitochondria and decreased in the cytoplasm from the 12 to $72 \mathrm{~h}$ in the BDL group, which was significantly different from that in the control and sham groups at the corresponding time points. Compared with the BDL group, Cytc expression levels in the cytoplasm for the BDL + Cytc group tended to gradually and significantly increase. Morphological changes in PMN mitochondria were marginal in BDL rats and marked in the BDL + Cytc group. In the BDL rats, $\mathrm{PMN}$ apoptosis was inhibited, a process induced by the mitochondrial apoptotic signaling pathway in which Cytc has an important role. High $\Delta \Psi \mathrm{m}$ in the mitochondria and decreased Cytc expression levels in the cytoplasm result in $\mathrm{PMN}$ apoptosis inhibition. Intravenous injection of Cytc may help compensate for the lack of Cytc proteins in the cytoplasm, inducing PMN apoptosis following BDL.

\section{Introduction}

Polymorphonuclear neutrophils (PMN) have an important role in host defense and various inflammatory diseases (1). Increased number, enhanced chemotaxis and superoxide anion generation of PMN have been observed in animal and clinical research with obstructive jaundice $(\mathrm{OJ})$, which is closely associated with exaggerated systemic inflammation (2-6). Several recent reports have focused on the fate of peripheral PMN and their significance in inflammatory diseases or surgical stress (7-11). Inhibited peripheral PMN apoptosis has been demonstrated in burns (7), severe trauma (8), systemic inflammatory response syndrome (SIRS) (9) and acute pancreatitis (10). The inhibition of PMN apoptosis may result in prolonged PMN life span in the circulation, leading to excessive inflammation (12). If the PMN function is infinitely exaggerated, it may lead to SIRS or even progress to multiple organ dysfunction syndrome (13).

The precise mechanisms underlying PMN apoptosis during OJ are poorly understood. Peripheral PMN have the shortest half-life among leukocytes (14). Senescent PMN undergo apoptosis, which is the principal signaling pathway responsible for cell destruction (15). It is thought that PMN apoptosis 
involves three signaling pathways (16): The endogenous pathway, the exogenous pathway and the endocytoplasmic reticulum pathway. These three signaling pathways communicate and interact with one another in physiological conditions, which has an important role in regulating PMN function and cell dissolution (17). Among the three signaling pathways, the passage from cytochrome $c$ (Cytc) to caspase cascade activation in the mitochondria is an important step that regulates cell death (18). Only $10 \mathrm{~min}$ are required to complete this process, which is termed the key ' 10 min event' (18). Therefore, the mitochondrial signaling pathway has an important role in controlling PMN apoptosis.

Recent studies reported that the outcome of acute inflammation in OJ is closely associated with the function of peripheral PMN (2-6). Previous studies demonstrated that delayed PMN apoptosis can cause excessive inflammation, and that the potential PMN apoptosis suppression mechanism may result in a decrease in caspase-3 activity $(19,20)$. It has been reported that caspase cascade activation is involved in the cell apoptotic signaling pathway, which has an impact on the initiation and outcome of inflammation (20). However, the endogenous mitochondrial apoptosis signaling pathway regulating cellular life-span and affecting PMN apoptosis in OJ has yet to be elucidated.

The present study used bile duct-ligated (BDL) rats to mimic OJ in order to investigate the changes in peripheral PMN apoptosis, the regulatory mechanism of the endogenous apoptotic signaling pathway, and the effect on PMN apoptosis following treatment with Cytc, as well as to attempt to elucidate the mechanisms underlying PMN apoptosis dysfunction in OJ.

\section{Materials and methods}

Animals. A total of 110 male Sprague-Dawley rats (weight, 200-250 g; age, 6-8 weeks) were purchased from the Medical Experimental Animal Center of Guangdong Province (Guangzhou, China), of which 104 survived. Food and water were provided ad libitum. The animals were housed in a laminar-flow specific pathogen-free atmosphere. A temperature of $22 \pm 1^{\circ} \mathrm{C}$ and $12 \mathrm{~h}$ light/dark cycle were maintained. All the experimental protocols were approved by the Animal Ethics Committee of the First Affiliated Hospital of Shenzhen University (Shenzhen, China).

Experimental design. The rats were randomly divided into four groups, of which the following numbers of rats survived: A control group $(n=8)$, a sham group $(n=32)$, a BDL group $(n=32)$, and a BDL + Cytc group $(n=32)$. The control group represented untreated rats at the beginning of the experiment. The remaining three groups were divided into rats at four time points: $12,24,48$ and $72 \mathrm{~h}$ following the BDL surgical procedure, with 8 rats at each time point group.

Prior to undergoing surgery the rats were subjected to a fast for $12 \mathrm{~h}$ with ad libitum access to water. All surgical procedures were performed under $10 \%$ chloral hydrate $(0.4-0.5 \mathrm{ml} / 100 \mathrm{~g}$; Sigma-Aldrich, St. Louis, MO, USA) intraperitoneal anesthesia with aseptic surgical techniques. Briefly, the common bile duct of the rats in the sham group was dissected away from the surrounding tissue but not ligated. In the BDL group and $\mathrm{BDL}+$ Cytc group, the surgical and postoperative care were the same as for the sham group. The rats in these two groups underwent BDL using double 4-0 silk sutures. Rats in the BDL + Cytc group were also injected intravenously with $20 \mathrm{mg} / \mathrm{kg}$ Cytc (Sigma-Aldrich) following BDL. The rats in the control group received no surgical procedure or treatment.

Sample collection. At the various time points prior to (in control rats) or following surgery (in experimental animals), all surviving animals were anesthetized with $10 \%$ chloral hydrate $(0.4-0.5 \mathrm{ml} / 100 \mathrm{~g})$, and the abdomen was opened prior to collection of blood samples from the inferior vein cava for biochemical analysis and PMN isolation. The rats were then sacrificed using cervical dislocation. The blood samples from each rat were sent to the Central Laboratory at the First Affiliated Hospital of Shenzhen University for analysis.

Liver function and blood routine examination. A total of $1 \mathrm{ml}$ of blood sample for each rat was centrifuged $(600 \mathrm{x} \mathrm{g}$, $10 \mathrm{~min}, 4^{\circ} \mathrm{C}$ ). The upper plasma was collected and assayed for total bilirubin (TBIL) and alanine aminotransferase (ALT) levels using a multi-channel automatic biochemical analyzer (Hitachi 7600; Hitachi, Ltd., Tokyo, Japan). A further $0.5 \mathrm{ml}$ of blood from each rat was used to count the number of blood cells using a three classification blood cell analyzer (ABX Hemastar-3; Horiba ABX SAS, Montpellier, France).

Isolation of peripheral PMN. In order to isolate peripheral PMN, $5 \mathrm{ml}$ blood samples were obtained from each rat. PMN were harvested using gradient centrifugation and hypotonic erythrocyte lysis, as described previously (21). The cells were centrifuged at $900 \mathrm{x}$ g for $10 \mathrm{~min}$ with cold RPMI-1640 medium (GE Healthcare Life Sciences, Logan, UT, USA) supplemented with $1 \%$ fetal bovine serum (Zhejiang Tianhang Biotechnology Co., Ltd., Huzhou, China). Giemsa staining (Amresco, LLC, Solon, OH, USA) and trypan blue (Sigma-Aldrich) exclusion test determined a purity of $>95 \%$ and a viability of $>98 \%$ of the PMN, respectively, in each sample.

PMN apoptosis. An Annexin V-fluorescein isothiocyanate (FITC) Apoptosis Detection kit (cat. no. FAK011.100; Neobioscience, Shenzhen, China) was used to detect early and late apoptotic activity following PMN isolation. According to the standard protocol, the binding buffer was diluted at 1:4 with sterile deionized water. A total of $50 \mu 1 \mathrm{PMN}$ suspension was resuspended with $150 \mu \mathrm{l}$ binding buffer, and $5 \mu \mathrm{l}$ Annexin V-FITC was added and gently mixed. Following an interval of $3 \mathrm{~min}, 10 \mu \mathrm{l}$ propidium iodide (PI; $20 \mu \mathrm{g} / \mathrm{ml}$ ) was added, incubated in the dark for $10 \mathrm{~min}$ following gentle mixing at room temperature, and then added to $250 \mu \mathrm{l}$ binding buffer prior to further mixing. The cells were detected and analyzed using a Fluorescence Activated Cell Sorter (FACS; Beckman Coulter Epics XL; Beckman Coulter, Inc., Brea, CA, USA). A total of $5 \times 10^{3}$ cells were counted, and Annexin- $\mathrm{V}^{+} / \mathrm{PI}^{-}$cells were indicative of apoptosis. The percentage of Annexin- $\mathrm{V}^{+} / \mathrm{PI}^{-}$cells was used to determine the apoptotic rate.

Measurement of PMN mitochondrial membrane potential $(\Delta \Psi \mathrm{m})$. A total of $10 \mu \mathrm{l}$ PMN suspension was washed twice with phosphate-buffered saline (PBS). The supernatant 
Table I. Serum ALT and TBIL biochemical levels at each time point.

\begin{tabular}{|c|c|c|c|c|c|c|}
\hline Plasma component & Groups & 0 & $12 \mathrm{~h}$ & $24 \mathrm{~h}$ & $48 \mathrm{~h}$ & $72 \mathrm{~h}$ \\
\hline \multirow[t]{4}{*}{ ALT, U/1 } & Control & $31.05 \pm 8.66$ & - & - & - & - \\
\hline & Sham & - & $35.10 \pm 8.65$ & $42.19 \pm 7.21$ & $24.64 \pm 6.36$ & $25.65 \pm 6.61$ \\
\hline & BDL & - & $62.75 \pm 10.82^{\mathrm{a}}$ & $57.25 \pm 6.67^{\mathrm{a}}$ & $52.38 \pm 10.56^{\mathrm{a}}$ & $49.95 \pm 8.08^{a}$ \\
\hline & $\mathrm{BDL}+\mathrm{Cytc}$ & - & $67.90 \pm 19.00^{\mathrm{a}}$ & $60.13 \pm 11.46^{\mathrm{a}}$ & $54.25 \pm 6.32^{\mathrm{a}}$ & $51.75 \pm 7.74^{\mathrm{a}}$ \\
\hline \multirow[t]{4}{*}{ TBIL, mg/dl } & Control & $0.14 \pm 0.06$ & - & - & - & - \\
\hline & Sham & - & $0.10 \pm 0.05$ & $0.08 \pm 0.05$ & $0.08 \pm 0.03$ & $0.09 \pm 0.04$ \\
\hline & BDL & - & $1.28 \pm 0.16^{\mathrm{a}}$ & $4.04 \pm 0.88^{\mathrm{a}}$ & $5.42 \pm 0.94^{\mathrm{a}}$ & $7.14 \pm 1.60^{\mathrm{a}}$ \\
\hline & $\mathrm{BDL}+\mathrm{Cytc}$ & - & $1.24 \pm 0.15^{\mathrm{a}}$ & $3.61 \pm 0.99^{\mathrm{a}}$ & $5.03 \pm 0.90^{\mathrm{a}}$ & $6.69 \pm 1.48^{\mathrm{a}}$ \\
\hline
\end{tabular}

Values are presented as means \pm standard deviation. ${ }^{\mathrm{a}} \mathrm{P}<0.01 \mathrm{vs}$. the control group and the sham group. ALT, alanine aminotransferase; TBIL, total bilirubin; BDL, bile duct-ligated; Cytc, cytochrome $c$.

was discarded and $20 \mu \mathrm{l}$ rhodamine-123 added (Rho-123; $10 \mu \mathrm{g} / \mathrm{ml}$; Sigma-Aldrich), incubated for $20 \mathrm{~min}$ at $37^{\circ} \mathrm{C}$, then washed twice in PBS for $5 \mathrm{~min}$. The cells were detected and analyzed using the FACS. A total of $5 \times 10^{3}$ cells were counted and the above procedures were repeated three times. Finally, the fluorescence intensity of the cells was detected by FACS using Rho-123, and the mean fluorescence intensity (MFI) was calculated.

Western blot analysis. Mitochondrial or cytosolic proteins from the PMN cells were extracted using a Cell Mitochondrial Isolation kit (Beyotime Institute of Biotechnology, Haimen, China), according to the manufacturer's protocol. They were then denatured in $5 \mathrm{X}$ sodium dodecyl sulfate (SDS)-loading buffer $(1,000 \mathrm{mmol} / 1$ Tris-HCL, $200 \mathrm{mmol} / 1$ dithiothreitol, $10 \%$ SDS, $1 \%$ bromophenol blue and 50\% glycerol; all components sourced from Sigma-Aldrich) at $100^{\circ} \mathrm{C}$ for $10 \mathrm{~min}$. Equal protein concentrations, determined using a bicinchoninic acid assay (Beyotime Institute of Biotechnology), were separated on $12 \%$ SDS-polyacrylamide gels (40 $\mu \mathrm{g} /$ lane) to detect Cytc in the subcellular fractions. Proteins were transferred to nitrocellulose membranes (Pierce Biotechnology, Inc., Rockford, IL, USA) and these were blocked in 5\% skim milk solution for $1 \mathrm{~h}$ at room temperature. Membranes were then incubated with rabbit primary antibodies at a dilution of 1:1,000, as follows, overnight at $4^{\circ} \mathrm{C}$ : Anti-Cytc (cat. no. 4272; Cell Signaling Technology, Inc., Danvers, MA, USA), anti-Cytc oxidase IV (a mitochondrial marker; cat. no. 4844; Cell Signaling Technology, Inc.) and tubulin (cat. no. 2128; Cell Signaling Technology, Inc.), to control for equal sample loading in each subcellular fraction. Membranes were washed in Tris-buffered saline with Tween 20, then incubated at room temperature for $1 \mathrm{~h}$ with horseradish peroxidase-conjugated goat anti-rabbit IgG (dilution, 1:2,000; BioVision, Inc., Milpitas, CA, USA). The membranes were then subjected to enhanced chemiluminescence (ECL) using an ECL detection kit (Pierce Biotechnology, Inc.), and quantified using Gel Pro Analyzer v. 4.0 software (Media Cybernetics, Inc., Rockville, MD, USA).

Transmission electron microscopy. Following PMN suspension centrifugation $\left(600 \mathrm{x} \mathrm{g}, 10 \mathrm{~min}, 4^{\circ} \mathrm{C}\right), 2.5 \%$ glutaraldehyde (Ted
Pella, Inc., Redding, CA, USA) was added to the precipitate and stored at $4^{\circ} \mathrm{C}$ overnight. The PMN were then dehydrated, saturated, embedded in Epon-812 epoxy resin (Ted Pella, Inc.), fixed using $1 \%$ osmic acid (Ted Pella, Inc.), dehydrated using an ethanol gradient (50, 70, 90 and 100\%), polymerized, sectioned with an EM UC7 ultramicrotome (Leica Microsystems GmbH, Wetzlar, Germany) and dyed using uranium acetate-lead citrate (Ted Pella, Inc.). Subsequently the sections were dried and images were captured using a transmission electron microscope (TEM; JEM-1400; JEOL, Ltd., Tokyo, Japan).

Statistical analysis. The data are presented as means \pm standard deviation. SPSS 17.0 (SPSS, Inc, Chicago, IL, USA) was used for to carry out the statistical analyses. Multiple comparisons were performed using One-way analysis of variance with Bonferonni's post-hoc test. $\mathrm{P}<0.05$ was considered to indicate a statistically significant value.

\section{Results}

Characteristics of the experimental models. The serum biochemical measurement values for the various groups are shown in Table I. ALT and TBIL levels in both the BDL and BDL + Cytc groups were significantly higher compared with those in the control group and the corresponding time point for the sham group $(\mathrm{P}<0.01)$. TBIL levels in the BDL and in the BDL + Cytc groups were significantly increased $12 \mathrm{~h}$ following the surgical procedure and remained $\geq 1.24 \mathrm{mg} / \mathrm{dl}$ throughout the subsequent experimental time points. The sham surgical procedure had no significant effect on TBIL levels. ALT levels in the BDL and in the BDL + Cytc groups were significantly increased compared with the control and sham groups $12 \mathrm{~h}$ after the surgical procedure, but decreased gradually in a time-dependent manner, reaching a minimum of $49.95 \mathrm{U} / 1$ throughout the subsequently observed time points.

Time-dependent changes in the number of white blood cells (WBC) and PMN following treatment with BDL and the Cytc. The WBC and PMN counts in each group, determined from blood sample collection, are presented in Table II. The WBC 
Table II. Time-dependent changes in the number of WBC and PMN at each time point.

\begin{tabular}{lcccccc}
\hline Blood cells & Groups & 0 & $12 \mathrm{~h}$ & $24 \mathrm{~h}$ & $48 \mathrm{~h}$ & $72 \mathrm{~h}$ \\
\hline WBC, $\mathrm{n} / \mu \mathrm{l}$ & Control & $5,081 \pm 2,150$ & - & - & - & - \\
& Sham & - & $5,250 \pm 2,162$ & $5,811 \pm 1,799$ & $5,679 \pm 1,996$ & $5,838 \pm 832$ \\
& BDL & - & $9,661 \pm 2,871^{\mathrm{a}}$ & $9,411 \pm 2,549^{\mathrm{a}}$ & $9,876 \pm 1,517^{\mathrm{a}}$ & $10,949 \pm 3,288^{\mathrm{a}}$ \\
& BDL + Cytc & - & $9,098 \pm 2,713^{\mathrm{a}, \mathrm{b}}$ & $8,986 \pm 1,250^{\mathrm{a}, \mathrm{b}}$ & $9,045 \pm 1,452^{\mathrm{a}, \mathrm{b}}$ & $9,481 \pm 2,706^{\mathrm{a}, \mathrm{b}}$ \\
PMN, $\mathrm{n} / \mu \mathrm{l}$ & Control & $488 \pm 258$ & - & - & - & - \\
& Sham & - & $570 \pm 88$ & $683 \pm 176$ & $673 \pm 212$ & $688 \pm 207$ \\
& BDL & - & $2,220 \pm 709^{\mathrm{a}}$ & $2,590 \pm 692^{\mathrm{a}}$ & $2,353 \pm 556^{\mathrm{a}}$ & $2,761 \pm 911^{\mathrm{a}}$ \\
& BDL + Cytc & - & $2,151 \pm 793^{\mathrm{a}, \mathrm{b}}$ & $2,300 \pm 817^{\mathrm{a}, \mathrm{b}}$ & $2,208 \pm 799^{\mathrm{a}, \mathrm{b}}$ & $2,460 \pm 420^{\mathrm{a}, \mathrm{b}}$ \\
PMN/WBC, & Control & $9.55 \pm 2.75$ & - & - & - & - \\
$\%$ & Sham & - & $13.51 \pm 7.51$ & $12.14 \pm 2.21$ & $13.88 \pm 9.00$ & $12.20 \pm 4.74$ \\
& BDL & - & $23.31 \pm 4.14^{\mathrm{a}}$ & $27.67 \pm 3.51^{\mathrm{a}}$ & $23.90 \pm 4.60^{\mathrm{a}}$ & $26.00 \pm 6.37^{\mathrm{b}}$ \\
& BDL + Cytc & & $23.80 \pm 4.46^{\mathrm{a}, \mathrm{b}}$ & $25.07 \pm 6.91^{\mathrm{a}, \mathrm{b}}$ & $24.63 \pm 8.58^{\mathrm{a}, \mathrm{b}}$ & $27.61 \pm 8.82^{\mathrm{a}, \mathrm{b}}$ \\
\hline
\end{tabular}

Values are presented as means \pm standard deviation ${ }^{\mathrm{a}} \mathrm{P}<0.01$ vs. control group and the sham group; ${ }^{\mathrm{b}}>0.05$ vs. BDL group. WBC, white blood cell; PMN, polymorphonuclear neutrophils; BDL, bile duct-ligated; Cytc, cytochrome $c$.

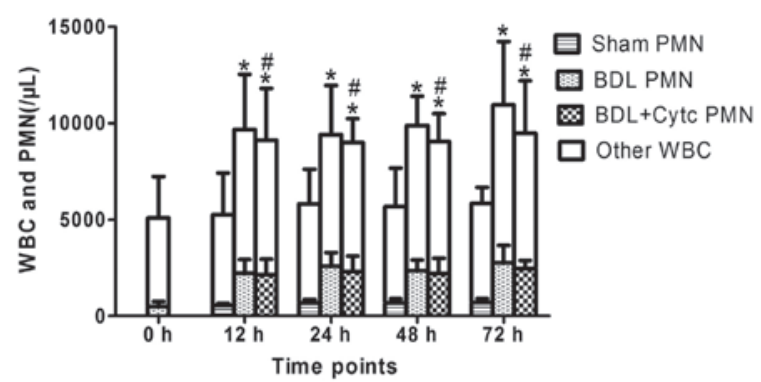

Figure 1. Time-dependent changes to PMN and WBC levels prior to and following the sham or BDL surgical procedure. PMN and WBC levels in the sham group remained low, displaying no significant difference compared with the control group. PMN and WBC levels in the BDL group increased gradually following the BDL surgical procedure. The BDL + Cytc group also displayed a similar increase in PMN and WBC levels, although this increase was not significant compared with the BDL group $\left({ }^{\#} \mathrm{P}>0.05\right)$. The error bars represent the mean \pm standard deviation. ${ }^{*} \mathrm{P}<0.01$ vs. the control group and the corresponding time point for the sham group. WBC, white blood cell; PMN, polymorphonuclear neutrophils; BDL, bile duct-ligated; Cytc, cytochrome $c$.

and PMN counts increased significantly $12 \mathrm{~h}$ following BDL, and continued to increase at 24, 48 and $72 \mathrm{~h}$ following BDL. The WBC and PMN counts revealed similar changes in the BDL + Cytc group, which were observed to be non-significantly different compared with the BDL rats at each time point $(\mathrm{P}>0.05)$. The sham surgical procedure had no significant effect on WBC and PMN count (Fig. 1).

Time-dependent changes in PMN apoptosis following BDL and treatment with Cytc. Sequential changes in PMN apoptosis at each time point are shown in Fig. 2. Significantly decreased PMN apoptotic levels were observed as early as $12 \mathrm{~h}$ following BDL, and these levels decreased further from $73.13 \pm 1.89 \%$ at $12 \mathrm{~h}$ to $64.93 \pm 3.24 \%$ at $72 \mathrm{~h}$ (Fig. 2). The values then remained unchanged until the end of the observation period. Compared

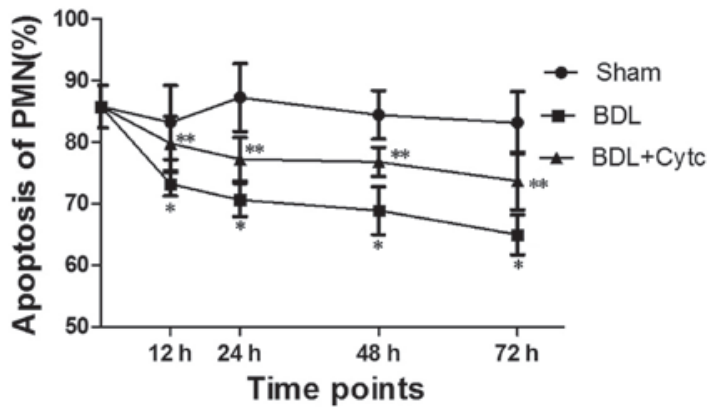

Figure 2. Time-dependent changes to PMN apoptosis levels, displayed as mean \pm standard deviation. ${ }^{*} \mathrm{P}<0.01$ vs. the control group and sham group. ${ }^{* *} \mathrm{P}<0.01$, vs. the BDL group at the corresponding time point. $\mathrm{PMN}$, polymorphonuclear neutrophils; BDL, bile duct-ligated.

with control group rats $(85.70 \pm 3.43 \%)$ and the sham rats at the respective time points, the PMN apoptosis rate of the BDL group was significantly decreased $(\mathrm{P}<0.01)$. Compared with the corresponding time points for the BDL group, the $\mathrm{BDL}+$ Cytc group showed a significantly increased PMN apoptosis rate $(\mathrm{P}<0.01)$.

Time-dependent changes in PMN $\triangle \Psi m$ following BDL and the Cytc administration on the mitochondrial apoptotic signaling pathway. Mitochondria have life-supporting functions and are able to regulate apoptosis (22-24). Cytc has a key role in the mitochondria, and participates in the assembly of a multimolecular complex known as the apoptosome, which is a core element of the apoptotic signaling pathway $(22,23,25)$. $\Delta \Psi \mathrm{m}$, which prevents apoptosis proteins (Cytc and so on) from being released from the mitochondria into the cytoplasm or nucleus, was detected using Rho-123 staining and FACS, and expressed as the MFI $(22,23)$. When the Rho-123 MFI value is lower, the $\Delta \Psi \mathrm{m}$ level is higher (26).

As shown in Fig. 3, Rho-123 MFI in PMN mitochondria was markedly decreased as early as $12 \mathrm{~h}$ following BDL, from 
A
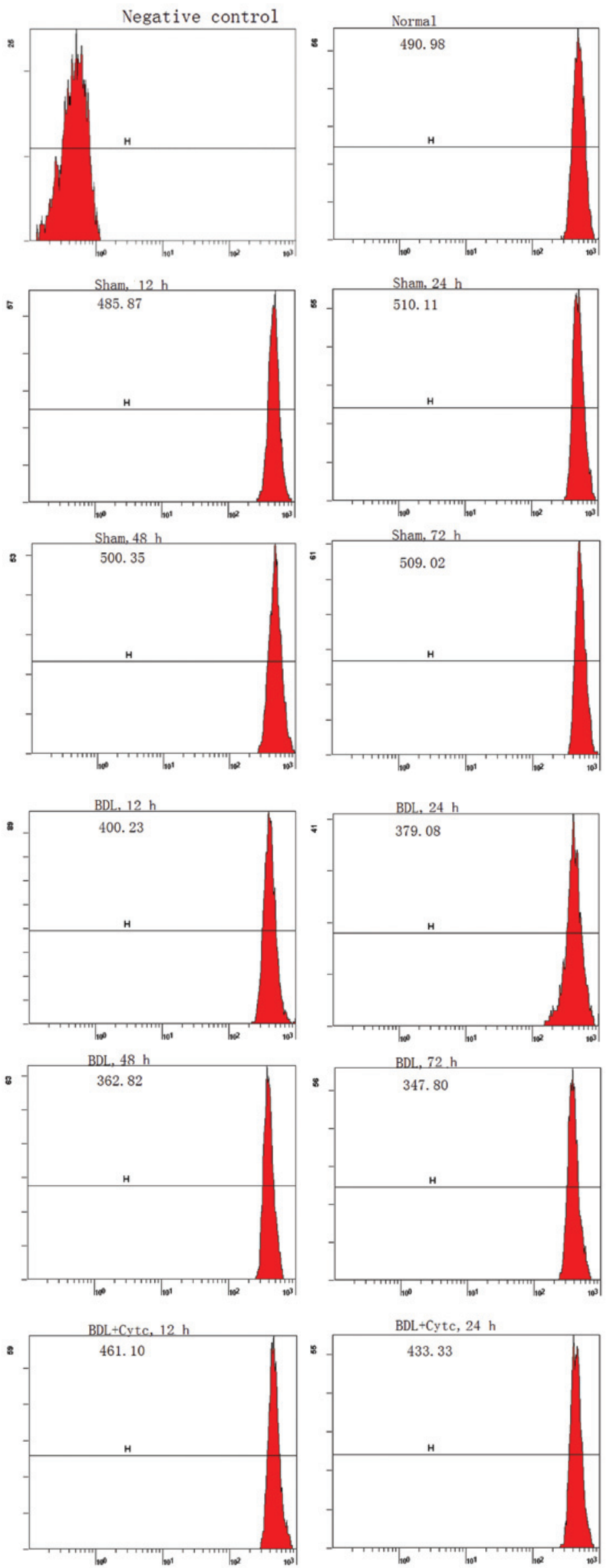

Figure 3. Time-dependent changes in PMN MFI. (A) PMN MFI was detected using Rho-123 staining on fluorescence-activated cell sorting. When the Rho-123 MFI value is lower, the $\Delta \Psi \mathrm{m}$ level is higher. Rho-123 MFI decreases in a time-dependent manner in the BDL group. However, the BDL + Cytc group displayed a gradual increase in MFI compared with the corresponding time points in the BDL group. 


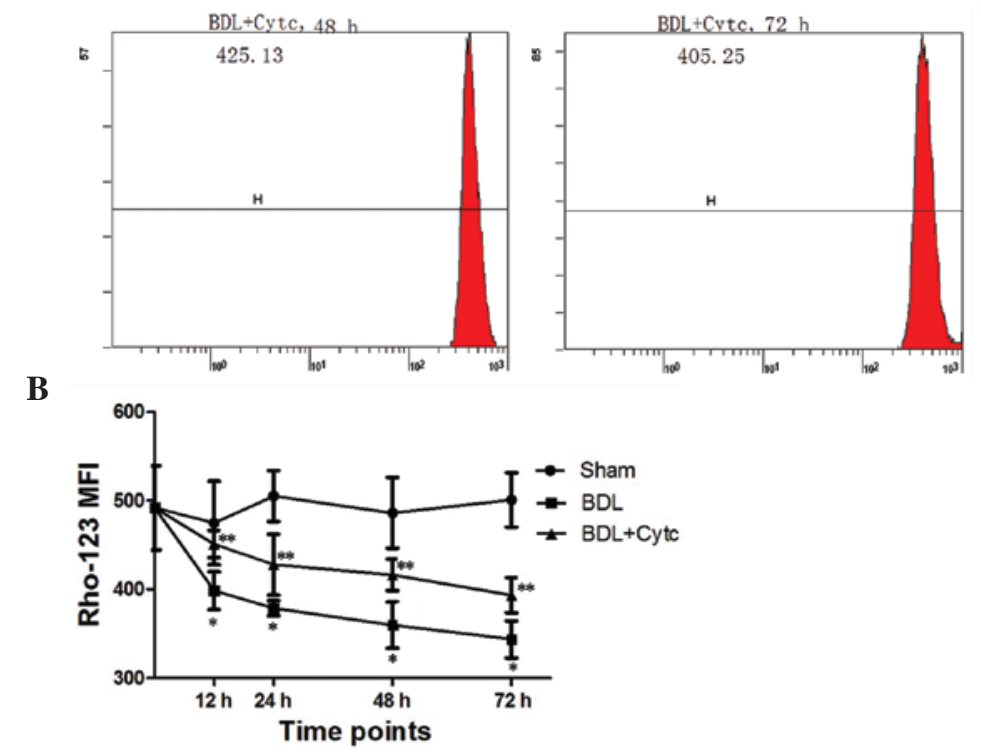

Figure 3. Continued. (B) The values are presented as the mean \pm standard deviation. ${ }^{*} \mathrm{P}<0.01$ and ${ }^{* *} \mathrm{P}<0.01$, vs. the control group and the corresponding time point of the sham group; ${ }^{* *} \mathrm{P}<0.01$, vs. the corresponding time point of the BDL group. PMN, polymorphonuclear neutrophils; MFI, mean fluorescence intensity; Rho-123, rhodamine-123; $\Delta \Psi \mathrm{m}$, mitochondrial membrane potential; BDL, bile duct-ligation; Cytc, cytochrome $c$.

$398.51 \pm 21.26$ at $12 \mathrm{~h}$ to $343.60 \pm 22.23$ at $72 \mathrm{~h}$, which then remained unchanged until the end of the observation period. Compared with the control group rats $(492.07 \pm 47.21)$ and the respective sham rat time points, the BDL group exhibited significant changes in MFI $(\mathrm{P}<0.01)$. Compared with the corresponding time point for the BDL group, the MFI in the $\mathrm{BDL}+$ Cytc group gradually increased $(\mathrm{P}<0.01)$.

Time-dependent changes in Cytc expression levels in the mitochondria and cytoplasm. Cytc expression levels in both the mitochondria and cytoplasm of the PMN are shown in Fig. 4. Cytc expression levels in the mitochondria increased in a time-dependent manner 12-72 $\mathrm{h}$ following BDL, and were markedly higher compared with the control group and the corresponding time point for the sham group. There were no differences in Cytc expression levels between the BDL and $\mathrm{BDL}+$ Cytc groups in the mitochondria (Fig. 4A,C). Cytc expression levels in the cytoplasm gradually decreased $12-72 \mathrm{~h}$ following BDL, and were markedly lower compared with that of the control group and the sham group at the corresponding time points. Cytc expression levels in the cytoplasm for the $\mathrm{BDL}+$ Cytc group gradually exceeded that of the BDL group at each time point (Fig. 4B and D).

Morphological changes in PMN mitochondria following $B D L$ and Cytc administration. The morphological changes in the PMN mitochondria were observed under TEM. As shown in Fig. 5, typical apoptotic changes in PMN, characterized by cell and nucleus shrinkage, retraction of pseudopodes, plasma membrane blebbing, chromatin condensation and nuclear fragmentation, were found in BDL rats, and markedly in BDL + Cytc rats. Furthermore, damaged structures, cracked cristae and vacuolization phenomena in the mitochondria were marginal in the BDL group, although more extensive in the BDL + Cytc group 12-72 $\mathrm{h}$ following the surgical procedure.

\section{Discussion}

Peripheral PMN is a type of acute inflammatory cell, the number and function of which impact inflammatory processes and their reversal, during the development of inflammatory diseases (27). Senescent PMN in the peripheral blood or infiltrating tissue undergo apoptosis. Apoptotic PMN are phagocytosed by macrophages without the release of proinflammatory mediators, leading to limited tissue injury and the end of inflammatory processes $(12,16)$. Inhibited peripheral PMN apoptosis has been demonstrated in burns (7), severe trauma (8), SIRS (9) and acute pancreatitis (10). The present study demonstrated that peripheral PMN apoptosis in rats was markedly inhibited $12 \mathrm{~h}$ following $\mathrm{BDL}$ and decreased in a time-dependent manner. The results also indicated that the number of PMN and WBC in BDL rats increased significantly, as compared with the control group rats and sham rats at each corresponding time point. Inhibited PMN apoptosis may result in increased numbers of PMN in the blood circulation, which benefits the host defense against systemic bacterial invasion, causing the uncontrolled release of toxic metabolites and leading to amplified systemic inflammation and organ injury $(10,28)$. It has been reported that cytoplasmic microinjection of Cytc promotes cell apoptosis activation (29). Accordingly, the present study used Cytc injection $(20 \mathrm{mg} / \mathrm{kg})$ in the tail vein of rats subjected to BDL to investigate the activating effect of Cytc on PMN apoptosis. The results demonstrated that the number of PMN decreased and PMN apoptosis increased. Compared with the corresponding time point for the BDL group, rats intravenously treated with Cytc following BDL exhibited a significant increase in the PMN apoptosis rate at 12, 24, 48 and $72 \mathrm{~h}$. It was therefore hypothesized that intravenous Cytc administration in rats subjected to BDL has an important role in inducing PMN apoptosis. The precise mechanism underlying this process requires further clarification.

Cell apoptosis is a complex process that has been the subject of numerous studies $(12,16,17)$. Apoptosis is a form of programmed 

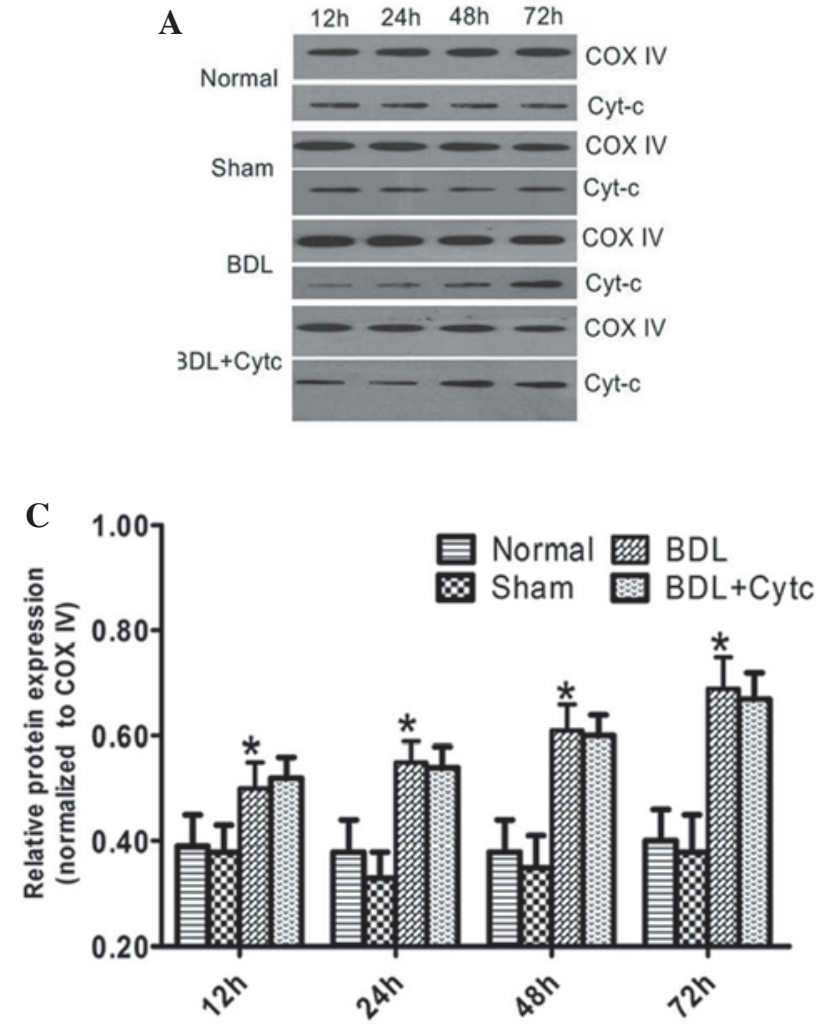

Cytc expression in the PMN mitochondria
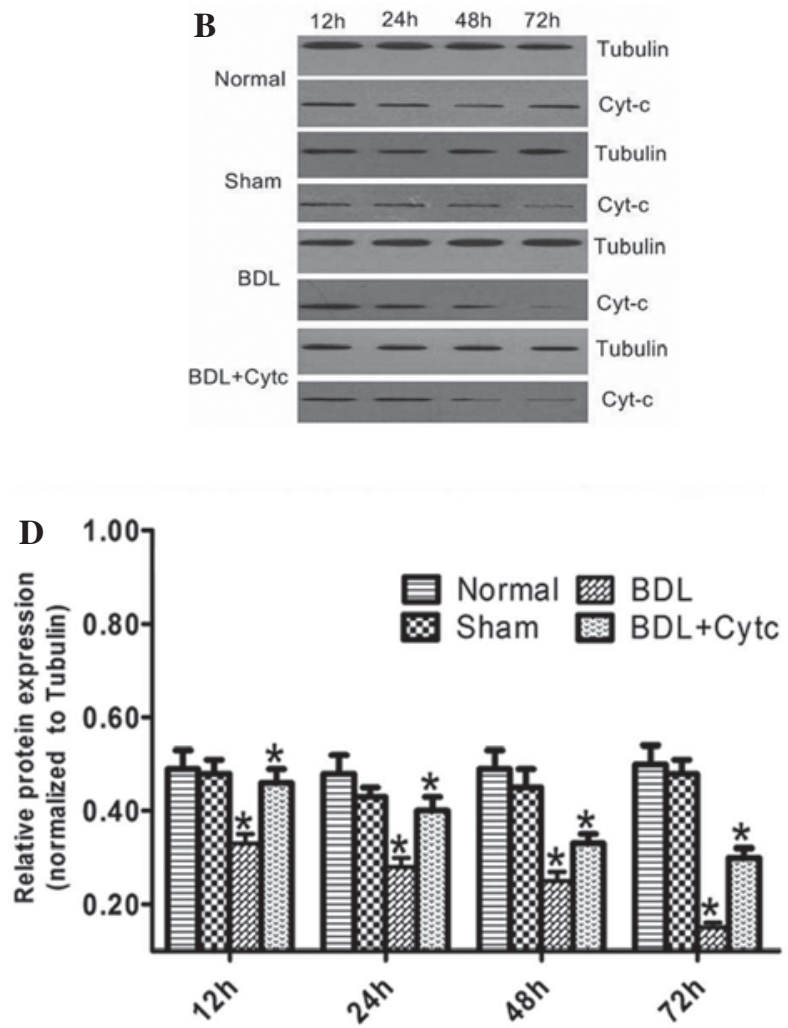

Cytc expression in the PMN cytoplasm

Figure 4. Western blots of Cytc expression in the PMN mitochondria and cytoplasm and graphs to reflect these data. Mitochondrial and cytosolic proteins were purified from the PMN at each time point and subjected to western blot analysis. (A) Cytc expression was increased in the mitochondria and (B) decreased in the cytoplasm from 12 to $72 \mathrm{~h}$ in the BDL group, which was significantly different from that in the control (normal) group and the sham group at the corresponding time points. Cytc expression in the cytoplasm for the BDL + Cytc group exceeded that of the BDL group at each time point. Control western blots demonstrate that the purified mitochondrial and cytosolic protein fractions were not contaminated with the mitochondrial membrane-bound protein COX-IV or the cytosolic protein $\beta$-tubulin. (C and D) Graphs to reflect these western blot analysis data. ${ }^{*} \mathrm{P}<0.01$ vs. control and sham groups. PMN, polymorphonuclear neutrophils; Cytc, cytochrome $c$; BDL, bile duct-ligation; COX-IV, Cytc oxidase-IV.

cell death, characterized by cell and nucleus shrinkage, retraction of pseudopodes, plasma membrane blebbing, chromatin condensation and nuclear fragmentation (30). This process is tightly controlled by gene regulation, receptor recognition and signal transduction (16). However, the potential mechanisms underlying PMN apoptosis have been extensively debated. It is thought that several factors have roles in this process and affect apoptosis development (22,31-34). Matsuda et al (11) showed that cytokine-modulated inhibition of PMN apoptosis at a local site augments PMN functions and induces excessive inflammatory response. The anti-apoptotic B cell lymphoma-2 (Bcl-2) family members Bcl-X-linked, A1 and myeloid cell leukemia-1, have been demonstrated to inhibit PMN apoptosis and promote survival by antagonizing the pro-apoptotic proteins (35). However, Fas ligation or ligation of the tumor necrosis-related apoptosis-inducing ligand receptor on PMN may also trigger apoptosis (36-38). Involved in either apoptosis or inflammation, caspases in PMN can be activated by intrinsically regulated apoptosis as well as death receptor-mediated apoptosis $(22,24)$. Despite a limited role of PMN mitochondria in cellular metabolism, certain investigations have suggested that these organelles may be involved in PMN cell death $(39,40)$. The results of the present study demonstrated that the mitochondria, although limited in number, do participate in PMN apoptosis in BDL rats. These organelles exhibit low mitochondrial enzymatic activity and do not synthesize a large amount of ATP, but preserve their $\Delta \Psi \mathrm{m}$ and contain proapoptotic proteins, which trigger PMN apoptosis if released into the cytosol (41). The mitochondria of the PMN were observed using TEM, demonstrating the presence of marginal morphological characteristic changes in BDL rats, and marked changes in BDL + Cytc rats, which exhibited damaged structures, cracked cristae and vacuolization phenomenon in the mitochondria.

Cytc is thought to be one of the most important pro-apoptotic proteins, and Cytc levels are markedly reduced in normal cell cytoplasm and markedly increased in apoptotic cells (22). $\Delta \Psi \mathrm{m}$ was detected using Rho-123 staining and FACS, and expressed as MFI to investigate the effect of Cytc on the mitochondrial apoptotic signaling pathway following BDL. The MFI value in BDL rats decreased from $415.66 \pm 30.77$ at $12 \mathrm{~h}$ to $388.51 \pm 31.66$ at $72 \mathrm{~h}$, a value that was significantly different compared with that of control group rats and sham rats at the corresponding time points. These results suggest that the $\Delta \Psi \mathrm{m}$ levels in PMN mitochondria increase gradually following BDL. Therefore, 

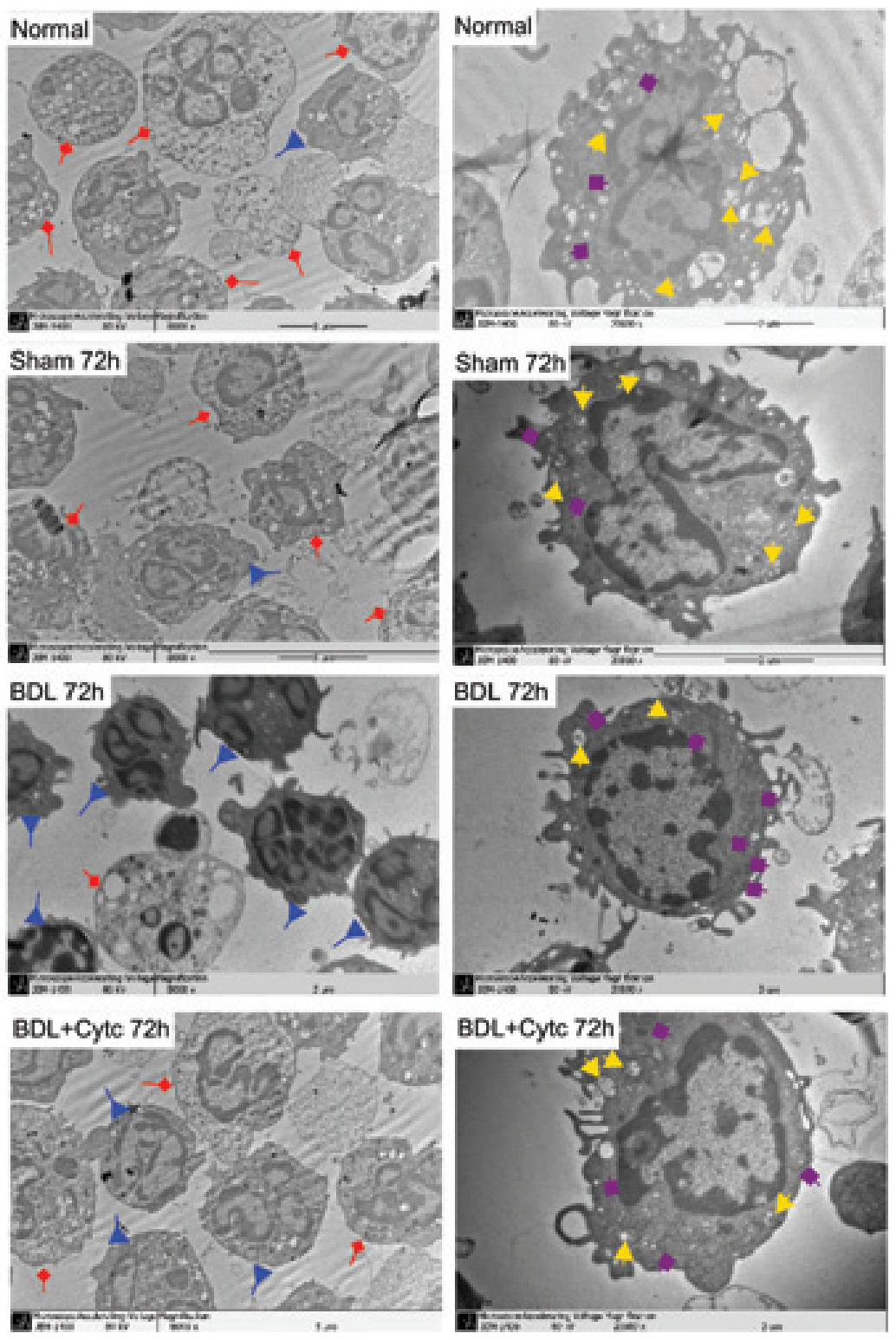

Figure 5. Morphological changes of mitochondrial PMN. Left panel, morphological changes of the PMN as determined by TEM (magnification, x8,000). Fewer apoptotic PMN and more normal PMN were observed at $72 \mathrm{~h}$ in the BDL group, but differing results were found in the control group and sham and BDL + Cytc groups at the corresponding time points, which displayed more apoptotic PMN and fewer normal PMN. Right panel, morphological changes of the PMN as determined by TEM (magnification, x20,000). Fewer damaged mitochondria and more normal mitochondria can be observed in the PMN of the BDL group at $72 \mathrm{~h}$, but differing results were found in the control group and sham and BDL + Cytc groups at the corresponding time points, which displayed more extensively damaged structures, cracked cristae and vacuolization of the mitochondria. Apoptotic PMN are represented in red; normal PMN are represented in blue; damaged mitochondria are represented in yellow; and normal mitochondria are represented in purple. PMN, polymorphonuclear neutrophils; TEM, transmission electron microscopy; BDL, bile duct ligation; Cytc, cytochrome $c$.

Cytc stored in the mitochondria did not move into the cytosol, inhibiting PMN apoptosis.

In an attempt to clarify the role of mitochondria in apoptosis, Cytc expression was investigated using western blotting. Cytc expression gradually increased in the mitochondria and decreased in the cytoplasm from 12 to $72 \mathrm{~h}$ in the BDL group, and these expression levels were significantly different from those of the control group and corresponding time points of the sham group. In the BDL + Cytc group Cytc was intravenously administered to the rats following BDL, demonstrating that the $\Delta \Psi \mathrm{m}$ of the PMN mitochondria gradually decreased and the Cytc expression levels in the cytoplasm increased. These results were significantly different from those of the BDL group at the corresponding time points. However, Cytc expression levels in the mitochondria remained elevated in the BDL + Cytc group from 12 to $72 \mathrm{~h}$, and these expression levels were not significantly different from those of the BDL group at the corresponding time points. The possible mechanisms underlying this phenomenon are as follows: i) The PMN may have spontaneously lost some of the Cytc proteins during the BDL process. ii) The $\Delta \Psi \mathrm{m}$ in the mitochondria is thought to be the conductor of the mitochondrial permeability transition pore. When the $\Delta \Psi \mathrm{m}$ decreases the pores open and Cytc proteins are released from the mitochondria into the cytoplasm (41). This suggests that Cytc expression levels gradually increase in the cytoplasm of the BDL + Cytc group from 12 to $72 \mathrm{~h}$. iii) Intravenous Cytc administration may directly infiltrate into the PMN cytoplasm, and has no 
significant effect on Cytc expression in the mitochondria. It is difficult to determine whether intravenous Cytc administration affects $\Delta \Psi \mathrm{m}$ in the mitochondria, and if so the exact amount of Cytc proteins that infiltrate into the cytoplasm via the intravenous route. Further studies are required in order to elucidate this mechanism.

In summary, the results of the present study suggested that PMN apoptosis is inhibited in BDL rats and the mitochondrial apoptotic signaling pathway participates in the apoptosis process. PMN mitochondria usually maintain their $\Delta \Psi \mathrm{m}$ within normal range. High $\Delta \Psi \mathrm{m}$ in the mitochondria and decreased Cytc expression levels in the cytoplasm may result in PMN apoptosis inhibition in BDL rats. Intravenous Cytc administration may help to compensate for the lack of Cytc proteins in the cytoplasm, inducing PMN apoptosis and reversal of inflammatory processes following BDL. These results provide an important theoretical basis for inflammatory complications during OJ.

\section{Acknowledgements}

The present study was supported by Science and Technology Planning Project of Guangdong Province (grant nos. 2012B031800349 and 2014A020212701), Medical Scientific Research Foundation of Guangdong Province (grant no. B2014326), Science and Technology Planning Project of Shenzhen (grant no. JCYJ20130401112153105), Medical Scientific Research Foundation of Shenzhen (grant no. 201401015).

\section{References}

1. Smith JA: Neutrophils, host defense, and inflammation: A double-edged sword. J Leuko Biol 56: 672-686, 1994.

2. Tsuji K, Kubota Y, Yamamoto S, Yanagitani K, Amoh Y, Takaoka M, Ogura M, Kin H and Inoue K: Increased neutrophil chemotaxis in obstructive jaundice: An in vitro experiment in rats. J Gastroenterol Hepatol 14: 457-463, 1999.

3. Takaoka M, Kubota Y, Tsuji K, Yamamoto S, Ogura M, Yanagitani K, Shimatani M, Shibatani N and Inoue K: Human neutrophil functions in obstructive jaundice. Hepatogastroenterology 48: 71-75, 2001.

4. Shibatani N, Yamamoto S, Kubota Y, Tsuji K, Takaoka M, Amoh Y, Matsushita M, Shimatani M, Imai Y and Inoue K: Neutrophil chemotaxis in bile duct-obstructed rats, and effect of internal biliary drainage. Hepatogastroenterology 49: 918-923, 2002.

5. Shimatani M, Tsuji K, Aze Y, Yamamoto S, Shibatani N, Imai Y, Takamido S, Kubota Y and Okazaki K: Effects of obstructive jaundice on neutrophil production and acquisition of chemotactic activity in the bone marrow. J Gastroenterol Hepatol 20: 117-125, 2005.

6. Badger SA, Jones C, McCaigue M, Clements BW, Parks RW, Diamond T, McCallion K and Taylor MA: Cytokine response to portal endotoxaemia and neutrophil stimulation in obstructive jaundice. Eur J Gastroenterol Hepatol 24: 25-32, 2012.

7. Chitnis D, Dickerson C, Munster AM and Winchurch RA: Inhibition of apoptosis in polymorphonuclear neutrophils from burn patients. J Leuko Biol 59: 835-839, 1996.

8. Ertel W, Keel M, Infanger M, Ungethum U, Steckholzer U and Trentz O: Circulating mediators in serum of injured patients with septic complications inhibit neutrophil apoptosis through up-regulation of protein-tyrosine phosphorylation. J Trauma 44 767-775; discussion 775-776, 1998

9. Fanning NF, Kell MR, Shorten GD, Kirwan WO, Bouchier-Hayes D, Cotter TG and Redmond HP: Circulating granulocyte macrophage colony-stimulating factor in plasma of patients with the systemic inflammatory response syndrome delays neutrophil apoptosis through inhibition of spontaneous reactive oxygen species generation. Shock 11: 167-174, 1999.
10. Chiu DF, Chen JC, Chen HM, Ng CJ, Shyr MH and Chen MF: Results of treating severe acute pancreatitis with gabexate is associated with neutrophil apoptosis activity. Hepatogastroenterology 50: 553-558, 2003.

11. Matsuda T, Saito H, Fukatsu K, Han I, Inoue T, Furukawa S, Ikeda $\mathrm{S}$ and Hidemura A: Cytokine-modulated inhibition of neutrophil apoptosis at local site augments exudative neutrophil functions and reflects inflammatory response after surgery. Surgery 129: 76-85, 2001.

12. Simon HU: Neutrophil apoptosis pathways and their modifications in inflammation. Immunol Rev 193: 101-110, 2003.

13. Maier RV: Pathogenesis of multiple organ dysfunction syndrome-endotoxin, inflammatory cells, and their mediators: Cytokines and reactive oxygen species. Surg Infect (Larchmt) 1: 197-204; discussion 204-205, 2000.

14. Colotta F, Re F, Polentarutti N, Sozzani S and Mantovani A: Modulation of granulocyte survival and programmed cell death by cytokines and bacterial products. Blood 80: 2012-2020, 1992.

15. Esmann L, Idel C, Sarkar A, Hellberg L, Behnen M, Möller S, van Zandbergen G, Klinger M, Köhl J, Bussmeyer U, et al: Phagocytosis of apoptotic cells by neutrophil granulocytes: Diminished proinflammatory neutrophil functions in the presence of apoptotic cells. J Immunol 184: 391-400, 2010.

16. Twomey $\mathrm{C}$ and McCarthy JV: Pathways of apoptosis and importance in development. J Cell Mol Med 9: 345-359, 2005.

17. Schwartz JT, Barker JH, Kaufman J, Fayram DC, McCracken JM and Allen LA: Francisella tularensis inhibits the intrinsic and extrinsic pathways to delay constitutive apoptosis and prolong human neutrophil lifespan. J Immunol 188: 3351-3363, 2012.

18. Green DR: Apoptotic pathways: Ten minutes to dead. Cell 121: 671-674, 2005.

19. Ottonello L, Frumento G, Arduino N, Bertolotto M, Dapino P, Mancini $M$ and Dallegri F: Differential regulation of spontaneous and immune complex-induced neutrophil apoptosis by proinflammatory cytokines. Role of oxidants, Bax and caspase-3. J Leukoc Biol 72: 125-132, 2002.

20. Le'Negrate G, Rostagno P, Auberger P, Rossi B and Hofman P: Downregulation of caspases and Fas ligand expression, and increased lifespan of neutrophils after transmigration across intestinal epithelium. Cell Death Differ 10: 153-162, 2003.

21. Nauseef WM: Isolation of human neutrophils from venous blood. Methods Mol Biol 412: 15-20, 2007.

22. Scorrano L: Opening the doors to cytochrome $c$ : Changes in mitochondrial shape and apoptosis. Int J Biochem Cell Biol 41: 1875-1883, 2009.

23. Gustafsson AB and Gottlieb RA. Heart mitochondria: Gates of life and death. Cardiovasc Res 77: 334-343, 2008.

24. Yang Y, Xing D, Zhou F and Chen Q: Mitochondrial autophagy protects against heat shock-induced apoptosis through reducing cytosolic cytochrome $c$ release and downstream caspase-3 activation. Biochem Biophys Res Commun 395: 190-195, 2010.

25. Mei Y, Yong J, Liu H, Shi Y, Meinkoth J, Dreyfuss G and Yang X: tRNA binds to cytochrome $\mathrm{c}$ and inhibits caspase activation. Mol Cell 37: 668-678, 2010.

26. Kurtoglu M and Lampidis TJ: From delocalized lipophilic cations to hypoxia: Blocking tumor cell mitochondrial function leads to therapeutic gain with glycolytic inhibitors. Mol Nutr Food Res 53: 68-75, 2009.

27. Fox S, Leitch AE, Duffin R, Haslett C and Rossi AG: Neutrophil apoptosis: Relevance to the innate immune response and inflammatory disease. J Innate Immun 2: 216-227, 2010.

28. Taneja R, Parodo J, Jia SH, Kapus A, Rotstein OD and Marshall JC: Delayed neutrophil apoptosis in sepsis is associated with maintenance of mitochondrial transmembrane potential and reduced caspase-9 activity. Crit Care Med 32: 1460-1499, 2004.

29. Kole AJ, Knight ER and Deshmukh M: Activation of apoptosis by cytoplasmic microinjection of cytochrome $c$. J Vis Exp pii; $2773,2011$.

30. Kroemer G, Dallaporta B and Resche-Rigon M: The mitochondrial death/life regulator in apoptosis and necrosis. Annu Rev Physiol 60: 619-642, 1998.

31. Lundqvist-Gustafsson H, Norrman S, Nilsson J and Wilsson A: Involvement of p38-mitogen protein kinase in Staphylococcus aureus-induced neutrophil apoptosis. J Leukoc Biol 70: 642-648, 2001.

32. Altavilla D, Saitta A, Squadrito G, Galeano M, Venuti SF, Guarini S, Bazzani C, Bertolini A, Caputi AP and Squadrito F: Evidence for a role of nuclear factor-kappaB in acute hypovolemic hemorrhagic shock. Surgery 131: 50-58, 2002. 
33. Sousa LP, Lopes F, Silva DM, Tavares LP, Vieira AT, Rezende BM, Carmo AF, Russo RC, Garcia CC, Bonjardim CA, et al: PDE4 inhibition drives resolution of neutrophilic inflammation by inducing apoptosis in a PKA-PI3K/Akt-dependent and NF-kappaB-independent manner. J Leukoc Biol 87: 895-904, 2010.

34. Fernandes CA, Fievez L, Ucakar B, Neyrinck AM, Fillee C, Huaux F, Delzenne NM, Bureau F and Vanbever R: Nicotinamide enhances apoptosis of G(M)-CSF-treated neutrophils and attenuates endotoxin-induced airway inflammation in mice. Am J Physiol Lung Cell Mol Physiol 300: L354-L361, 2011.

35. Paunel-Görgülü A,Zörnig M,Lögters T, Altrichter J, Rabenhorst U, Cinatl J, Windolf J and Scholz M: Mcl-1 mediated impairment of the intrinsic apoptosis pathway in circulating neutrophils from critically ill patients can be overcome by Fas stimulation. J Immunol 183: 6198-6206, 2009.

36. Nwakoby IE, Reddy K, Patel P, Shah N, Sharma S, Bhaskaran M, Gibbons N, Kapasi AA and Singhal PC: Fas-mediated apoptosis of neutrophils in sera of patients with infection. Infect Immun 69: 3343-3349, 2001.
37. Dunican AL, Lenemoth SJ, Grutkoski P, Ayala A and Simms HH: TNF alpha-induced suppression of PMN apoptosis is mediated through interleukin-8 production. Shock 14: 284-288; discussion 288-289, 2000

38. Geering B and Simon HU: A novel signaling pathway in TNF $\alpha$-induced neutrophil apoptosis. Cell Cycle 10: 2821-2822, 2011.

39. Maianski NA, Mul FP, van Buul JD, Roos D and Kuijpers TW: Granulocyte colony-stimulating factor inhibits the mitochondria-dependent activation of caspase-3 in neutrophils. Blood 99: 672-679, 2002.

40. Maianski NA, Roos D and Kuijpers TW: Tumor necrosis factor alpha induces a caspase-independent death pathway in human neutrophils. Blood 101: 1987-1995, 2003.

41. Maianski NA, Geissler J, Srinivasula SM, Alnemri ES, Roos D and Kuijpers TW: Functional characterization of mitochondria in neutrophils: A role restricted to apoptosis. Cell Death Differ 11: 143-153, 2004. 